\title{
System to Estimate Ages and Redshifts for Radio Galaxies
}

\author{
O. V. Verkhodanov, A. I. Kopylov, N. V. Verkhodanova, O. P. \\ Zhelenkova, V. N. Chernenkov, Yu. N. Parijskij, N. S. Soboleva, A. V. \\ Temirova
}

Special Astrophysical Observatory, Nizhnij Arkhyz, Russia

\begin{abstract}
The system allowing a user to operate at the server sed.sao:ru with simulated curves of spectral energy distributions (SED) and to estimate ages and redshifts from photometric data is described.
\end{abstract}

The existence of a huge volume of observational data in the optical and infrared wavelength range increases significantly our knowledge about the distant Universe. However, information about the space distribution of extragalactic objects is not yet accessible because of the limits of observational possibilities: the direct measurement of redshifts is possible with spectroscopic methods having a sensitivity 2 magnitudes lower than photometric ones. Special interest lies in the study of distant objects allowing astronomers to investigate both largescale structures andthe evolution of active galactic nuclei (AGNs) (which are connected with black holes). Spectroscopy of such objects is rather difficult. However, using photometric data one can essentially simplify this problem since it allows an astronomer to make the initial selection.

To accelerate a procedure of age (and photometric redshift) estimation we have begun a project "Evolution of radio galaxies", which has to allow a user to obtain age and photometric redshift estimates. The main tasks of the system are: 1) estimate of ages with fixed redshift $z ; 2$ ) estimate of both ages and $z$; 3) archiving of optical observations of RC radio galaxies (in FITS, JPEG, PS formats with text comments); 5) archiving of the main publications by the current topic; 6) development of HTPP and e-mail access; 7) local SED operation to simulate an observational process.

To estimate ages and redshifts with photometric data we operate with simulated curves of spectral energy distributions (SED) for different types of galaxies of two models PEGASE (Fioc, Rocca-Volmerange, 1997, 1999), GISSEL96 (Bruzual, Charlot, 1993; Bolzonella et al., 2000).

Before the estimate of values we smooth a SED with a filter transmission curve to simulate observational data using a "compressing" filter with the growth of redshift: $S_{i k}=\frac{\sum_{j=0}^{n} s_{i-n / 2+j} f_{j k}(z)}{\sum_{j=0} f_{j k}(z)}$. Here $s_{i}$ is the initial synthetic SED, $S_{i k}$ is the smoothed SED in the $k$-th filter, $f_{k}(z)$ is the transmission in the k-th filter, "compressed" by $(1+z)$ times "moving" along the SED, $j=1, n$ is the pixel index in the curve of filter transmission.

The estimate of ages and redshifts is performed by selection of the optimum location on the SED curves of the measured photometric points obtained when observing radio galaxies in different filters. We use the already computed 
tabulated SED curves for different ages. Using discrepancies we construct a probability function in the form of $p=\frac{1}{\max } \exp \left(\chi^{2}\right)$, where $\max$ is the maximum value of the calculated function. $\chi$ is the discrepancy calculated by sliding the photometric points along the SED curve:

$$
\chi^{2}=\sum_{k=1}^{N \text { filters }}\left(\frac{F_{o b s, k}-p S E D_{k}(z)}{\sigma_{k}}\right)
$$

Here $F_{o b s, k}$ is the observational magnitude in the $k$-th filter, $\operatorname{SED}_{k}(z)$ is the simulated magnitude for the given SED in the $k$-th filter at the given redshift $z$, $p$ is the free coefficient, $\sigma_{k}$ is the error of the observed magnitude.

In order to take account of the absorption, we apply the maps (as FITS-files from the paper "Maps of Dust IR Emission for Use in Estimation of Reddening and CMBR Foregrounds" (Schlegel et al., 1998).

The system is situated on the special Web-server http://sed.sao.ru operating in the Linux Red Hat (6.2) system, unifying various type resources and accessed by FTP, HTTP and e-mail. Typical e-mail form for a request looks like this:

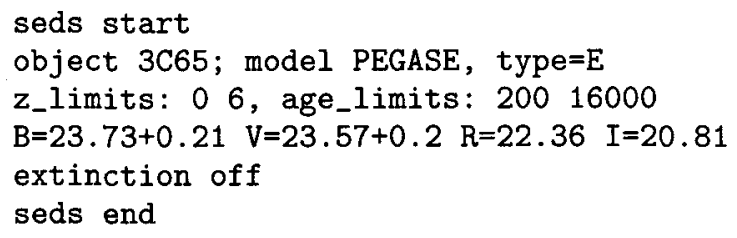

Here seds start and seds end are opening and closing keywods of the form. Keywords object, model and type determine an object name, a type of a model and a type of a galaxy, respectively. Available galaxy types are E, S0, Sa, Sb, Sc, Sd. z_limits and age_limits determine the limits of search for a redshift and an age (in Myr). The observed magnitudes age given with $\mathrm{B}, \mathrm{V}, \mathrm{R}, \mathrm{I}, \mathrm{J}, \mathrm{H}, \mathrm{K}, \mathrm{g}$, $r$, i, etc. keywods corresponding to the filter names. The error of the magnitude detection is given via plus ' + ' after a value of the magnitude. Extinction in this example is not calculated.

Other supported possibilities are a) sorted bibliographic collection of papers for different stages of radio galaxy evolution; b) archive of radio galaxy data in various wavelength ranges (both observed in the Special Astrophysical Observatory and taken from the Internet), containing information on the objects and figures in FITS, JPEG and PostScript formats.

The project was supported by the Russian Foundation for Basic Research (Grant No 99-07-90334).

\section{References}

Bolzonella M., Miralles J.-M., Pelló R. 2000. Astron. Astroph., 363, 476

Bruzual G., Charlot S. 1993, ApJ, 405, 538

Fioc M., Rocca-Volmerange B., 1997, Astron. Astroph., 326, 950, 1999: astro$\mathrm{ph} / 9912179$

Schlegel, D., Finkbeiner, D., Davis, M., 1998, ApJ, 500, 525 